\title{
Kochende Medien: (Trans-)Regionalität, (Trans-)Nationalität und (Trans-)Kulturalität im Kontext televisueller Koch-Formate York Kautt
}

Televisuelle Koch-Formate ${ }^{1}$ sind weltweit verbreitet. In Asien, Nord- und Südamerika, in Afrika, Australien und Europa - in den globalen Massenmedien wird massenhaft gekocht. Wenngleich für die vorliegende Untersuchung keine Zahlen ermittelt wurden, die etwa Rückschlüsse über die Popularität und die quantitative und qualitative Differenzierung von Formaten in einzelnen Regionen zuließen, lässt sich die Verbreitung televisueller Koch-Formate als solche unter den gegebenen Internetbedingungen schnell verifizieren. ${ }^{2}$ Blickt man auf die Situation in der BRD, lässt sich der Erfolg von Koch-Formaten - gemessen an den Sehbeteiligungen und der in den letzten Jahren deutlich angestiegenen Anzahl von Formaten - hingegen eindrucksvoll belegen. ${ }^{3}$ Für vorliegende Untersuchung ist jedoch weniger die Frage nach dem faktischen Erfolg der Koch-Formate auf der Seite der Rezipienten bedeutsam. Die hier behandelte Frage ist vielmehr diejenige, unter welchen Gesichtspunkten man von einer Transnationalisierung televisueller Koch-Formate sprechen kann und im Rahmen welcher Strukturen Transnationalisierungsprozesse überhaupt ermöglicht und forciert werden.

1 Mit dem Begriff des Formats wird hier eine Sendeeinheit bezeichnet, die über die Zuordnung von Sender und Sendezeit sowie über die Beibehaltung von Erscheinungsformen im weitesten Sinne (Skript, Bühnen, Vor- und Abspann, Rollendifferenzierung, Schnitt, Lichtsetzung, Kameraführung u. a.) eine wiedererkennbare Form annimmt.

2 Die Internetrecherche zeigt zugleich, dass sich neben den professionell produzierten TV-KochFormaten in den verschiedensten Weltregionen eine nicht zu überschauende Vielzahl privat produzierter Darstellungsformen von Kochhandlungen etabliert hat (Blogs, Newsgroups, Homepages), in denen Fotos und Filme zum Einsatz gebracht werden.

3 Bei den in Prozent gemessenen Marktanteilen (MA), die im Unterschied zu den absoluten Zahlen der Sehbeteiligten (gemessen in Millionen Zuschauern) Auskunft über das Auswahlverhalten der Rezipienten vor dem Hintergrund sämtlicher Alternativen im jeweiligen Zeitrahmen geben, zeigt sich z. B. für elf erfasste Formate des Jahres 2008 ein durchschnittlicher MA von 5,93 Prozent bei Spitzenwerten von 13,3 Prozent („Lafer! Lichter! Lecker!“‘, 08.11.08, ZDF). Diese Berechnungen basieren auf Zahlen, die vom Autor von der Gesellschaft für Konsumforschung (GfK Fernsehforschung) bezogen wurden. In den Jahren 2007 bis 2009 waren im deutschsprachigen Fernsehen kontinuierlich etwa dreißig Serien-Formate etabliert. In der Woche 04.05.2009 bis zum 10.05.09 wurden z. B. täglich überschneidungsfrei durchschnittlich 3,5 Stunden Koch-Formate gesendet, wobei der quotenwichtige Freitag mit mehr als 7,5 Stunden den Spitzenwert erreicht (siehe „tvtv“ vom 30.04.2009). 


\section{Kochformate und/als transnationale Medienproduktionen}

Vor jeder empirischen Analyse der Koch-Formate auf der Ebene ihrer Inszenierungen kann man feststellen, dass die Transnationalisierung der Koch-Formate maßgeblich mit der Funktionsweise des Systems der Massenmedien zu tun hat, wobei für Letzteres die Wirtschaft eine wesentliche Rolle spielt. Denn in den meisten Weltregionen operieren Medienanbieter auch als Wirtschaftsunternehmen, die sich auf Wirtschaftsmärkten in Konkurrenzumgebungen behaupten müssen. Einschaltquoten müssen zu Zwecken der Steigerung von Werbeeinnahmen maximiert, Produktionskosten minimiert werden. So sind die folgenden Ausprägungen einer transnationalen Medien-Koch-Kultur fraglos (auch) der Rationalität der Wirtschaft geschuldet: A) Formate werden für die Ausstrahlung in verschiedenen Ländern lediglich sprachlich adaptiert, so z. B. das britische Format „Oliver's Twist“ (ausgestrahlt in 70 (!) Ländern), das japanisches Format „Ryori no Tetschuschin“ bzw. „Iron Chef“ (ausgestrahlt in den USA und England) oder das US-amerikanische Format „Bizarre Foods“ (USA), das in Deutschland unter dem Namen „Krasse Küche“ läuft. B) Anbieter kopieren und modulieren Skripts, die bereits andernorts etabliert sind (so dient z. B. das Konzept des britischen Formats „Come dine with me“ (Erstausstrahlung 2005), in mehreren Ländern (u. a. Türkei, Belgien, Iran, Ungarn, Spanien, Russland) als Vorlage, wobei die deutsche Modulation unter dem Namen „Das perfekte Dinner“ produziert wird. C) Einzelne Sender präsentieren innerhalb ihres Sendeplatzes ein Spektrum von Formaten unterschiedlicher Herkunftsregionen (so zeigt etwa das US-amerikanische Food Network britische und japanische Formate). D) Televisuelle Koch-Formate werden systematisch intertextuell mit Formen eines transnationalen Marketings verknüpft, sodass beide Seiten (Kochformate/Werbung) für die Wirtschaft als weithin sichtbare Bühnen fungieren, die wechselseitig aufeinander bezogen werden können.

Die genannten Produktionsstrategien unterliegen einem wirtschaftlichen Kalkül, dessen Ziel der ökonomischen Gewinnmaximierung nicht weiter erläutert werden muss. Nun ist mit dem Hinweis auf die Interessen der Wirtschaft aber noch nicht erklärt, wie es den Koch-Formaten als Kommunikationen gelingt, zu transnationalen Phänomenen zu werden, ja gleichsam zu Erfolgsmedien für die Massenmedien. Diese Frage soll im Folgenden im Zugriff auf die Medientexte selbst genauer in den Blick genommen werden. 


\section{Technische Bilder und die transnationale/transkulturelle Anreizstruktur televisueller Koch-Formate}

Die Frage nach dem (Nicht-)Erfolg einzelner Kochformate kann auf der Ebene der Akteure nur im Rahmen von Rezeptionsstudien ergründet werden, die die individuellen Motiv- und Interessenlagen als solche rekonstruieren. Im Rahmen entsprechender Untersuchungsdesigns lässt sich auch klären, inwiefern situative Kontexte und verschiedene Modi der Vergemeinschaftung (Klassenlage, Milieu, Lebensstil, Szene) für die Bewertungen einzelner Formate und Sendungen durch die individuellen Akteure von Bedeutung sind. Hier geht es indessen um die Frage, welche Anreize die Kommunikationen in sich selbst herstellen, um ihre Akzeptanz wahrscheinlicher zu machen und inwiefern eben dadurch potentielle Voraussetzungen für die Transnationalisierung der Formate geschaffen werden. Zu diesem Zweck werden im Folgenden die Ergebnisse einer empirischen Analyse der Medientexte vorgestellt. Den materialen Korpus der Untersuchung bildet eine Erhebung von 21 Formaten (vgl. Tabelle 1), die fast alle im deutschen Fernsehen zwischen 2007 und 2009 zu sehen waren, aber auch in verschiedenen Ländern des inner- und außereuropäischen Raumes ausgestrahlt wurden. ${ }^{4}$

Tabelle 1: Erhobene Koch-Formate

\begin{tabular}{|l|l|l|l|}
\hline \multicolumn{1}{|c|}{ Format-Name } & \multicolumn{1}{|c|}{ Datum } & \multicolumn{1}{|c|}{ Sender } & \multicolumn{1}{c|}{ Nation } \\
\hline $\begin{array}{l}\text { „Anthony Bourdain: A Cook's } \\
\text { Tour“ }\end{array}$ & 16.11 .08 & DMAX & USA \\
\hline „Gordon Ramsays Höllenküche“ & 16.11 .08 & DMAX & GB \\
\hline „Oliver's Twist“ & 15.11 .08 & RTL2 & GB \\
\hline „La Cocina de Biachi“ & 02.08 .10 & Canal 1 & Ecuador \\
\hline „Krasse Küche“ & 09.11 .08 & RTL2 & USA \\
\hline „Iron Chef“ & 12.03 .00 & Fuji TV & Japan \\
\hline „Lafer! Lichter! Lecker!“ & 25.06 .00 & Food Net & \\
\hline „J.B. Kerner“ & 08.11 .08 & ZDF & BRD \\
& 05.09 .09 & & \\
\hline „Alfredissimo“ & 09.11 .07 & ZDF & BRD \\
& 16.11 .08 & & BRD \\
\hline
\end{tabular}

4 Unter computerisierten Medienbedingungen ist die Verfügbarkeit einzelner Sendungen zudem nicht mehr auf terrestrische Sendegebiete beschränkt - insbesondere das Internet trägt zur Transnationalisierung einzelner Formate jenseits von TV-Ausstrahlungen bei. 


\begin{tabular}{|l|l|l|l|}
\hline „Die Kochprofis - Einsatz am & 08.11 .08 & RTL 2 & BRD \\
Herd“ & 30.11 .09 & & \\
\hline „Das perfekte Dinner“ & 09.11 .08 & VOX & BRD \\
& 24.11 .09 & & \\
\hline „Lafer: Einfach kochen“ & 05.12 .07 & EinsPlus & BRD \\
\hline „Ganz und Gar Henssler“ & 09.11 .07 & VOX & BRD \\
\hline „Silent Cooking“ & 03.12 .07 & VOX & BRD \\
\hline „Die Kocharena“ & 08.11 .07 & VOX & BRD \\
\hline „Schuhbeck's“ & 11.11 .07 & BR & BRD \\
\hline „Die Kochprofis - Hausbesuch“ & 08.12 .07 & RTL2 & BRD \\
\hline „Die Küchenschlacht“ & 10.11 .08 & ZDF & BRD \\
\hline „Polettos Kochschule“ & 23.11 .09 & & \\
\hline „Mein Restaurant“ & 09.11 .08 & NDR & BRD \\
\hline „Essen und Trinken“ & 11.11 .08 & VOX & BRD \\
\hline
\end{tabular}

Quelle: Eigene Darstellung.

Das methodische Vorgehen der empirischen Untersuchung folgt einer Richtung der qualitativen Sozialforschung, die die Kategorien zur Erklärung und Beschreibung der jeweiligen Gegenstände nicht an die Materialien herantragen, sondern aus deren Analyse selbst gewinnen will. Insofern orientiert sich die Untersuchung an Methoden wie an der von Glaser und Strauss sogenannten und von diesen geprägten „grounded theory“ (z. B. Glaser/Strauss 1967). Indem sie die Frage nach dem Erfolg der Formate in den Mittelpunkt stellt, schränkt sie die Möglichkeiten der Theoriebildung jedoch systematisch ein. An die Stelle der Frage ,Was geht hier eigentlich vor?" und der damit zusammenhängenden ,Leitkategorie‘ des Untersuchungsgegenstandes tritt die Frage nach denjenigen Merkmalen der Koch-Formate, die deren Attraktivität potentiell zu steigern vermögen. Die Kommunikate selbst werden dabei als Texte aufgefasst, die entlang audiovisueller Differenzen Sinnformen bilden, die sich sinnverstehend rekonstruieren lassen. ${ }^{5}$ Das Analyseverfahren basiert im Wesentlichen auf Instrumenten der Filmanalyse, die bei der Interpretation den Einsatz von Sprache, Musik und Geräuschen ebenso berücksichtigt wie die filmischen Gestaltungstechniken (Einstellungsgröße, Einstellungsdauer, Kameraperspektive u. a.).

Die folgende Darstellung der Anreizstruktur der Koch-Formate wird hier aus Platzgründen auf einige zentrale Dimensionen eingeschränkt.

5 Zum Film als einem filmsprachlichen wie filmbildnerischen Zeichensystem siehe exemplarisch Keppler 1985: 43-90. 


\subsection{Universalität und Sperifikation}

Essen und Kochen sind universale Themen, die jedem Menschen aus dem je eigenen Erleben, Handeln und Kommunizieren vertraut sind. Nun macht die Vertrautheit der Rezipienten mit dem Thema die Attraktivität der Koch-Formate für sich allein genommen nicht wahrscheinlicher. Vor dem Hintergrund einer oftmals für unabdingbar gehaltenen Orientierung am Neuen und Innovativen der Massenmedien spricht die Präsentation des Bekannten sogar eher für Publikumsreaktanz. Entscheidend ist es daher zu sehen, dass die Universalität des Themas nicht mit einer redundanten Behandlung desselben gleichzusetzen ist. Die gezeigten Bilder machen vielmehr unmittelbar und von Moment zu Moment immer wieder aufs Neue evident, dass das Allgemeine und Bekannte im jeweiligen Einzelfall als das Besondere und Unbekannte in Erscheinung tritt. Dieser Sachverhalt gilt auch für die Formen des Essens und Kochens jenseits der Massenmedien, gewinnt aber in den televisuellen Bilder-Rahmen, die die Gestaltwerdung der Speisen in ihrer Verlaufsform präsentieren, eine besondere Bedeutung. Ein grundlegender narrativer Spannungsbogen der Koch-Formate muss also in keinem Drehbuch fixiert werden. Er ergibt sich dadurch, dass die Speise vor dem Hintergrund von Wissensbeständen und Erfahrungen als das Besondere Form annimmt, wobei die Relation von bekannten zu unbekannten Details mit dem individuellen Vorwissen der Rezipienten variiert.

\subsection{Anschaunngsreize}

Die von Plessner so genannten Zustandssinne (Tast-, Geschmacks- und Geruchssinn), die für die Alltagspraxis des Essens von großer Bedeutung sind, können von den Koch-Formaten nicht unmittelbar angesprochen werden. Der Alltagsweisheit ,Die Augen essen mit kann hingegen im Rahmen einer an die Sinne appellierenden Bildsprache durchaus folgenreich entsprochen werden. Von einer visuellen, Ästhetisierung ' des Essens lässt sich schon deshalb sprechen, weil der Rahmen der Bilder, im Unterschied zur bloßen Wahrnehmung des Sehsinns mit seinen weniger scharf markierten Grenzen des Sehfeldes, die sichtbaren Erscheinungsformen innerhalb des Rahmens betont. Deutlich wird das insbesondere in den als Höhepunkte inszenierten Momenten, in denen Arrangements fertig zubereiteter Gerichte auf Tellern gezeigt werden.

Nun trägt die bildliche Vorführung ästhetisch präparierter Speisen aber nicht nur zur Transnationalisierung bei, indem die Adressierung der physischen Sinne kulturabhängige Sinnstrukturen unterläuft. Die in verschiedenen Sprachräumen existierende Wissensformel ,Die Augen essen mit“ ist vielmehr als eine „Sollensformel einer Kultur der Gastlichkeit“ zu begreifen, die eine „Basis des Zusammenlebens“ 
schafft (Wierlacher 2005: 138). In der Arbeit an der schönen Form kommt - auch in den televisuelle Koch-Formaten - nicht nur die Kreativität der Köche, sondern auch die rituelle Dimension der Wertschätzung der an der Mahlzeit Teilnehmenden zum Ausdruck.

\subsection{Sinnvolles Handwerk, professionelle Könnerschaft und Kreativität}

In sämtlichen Formaten lassen die Bilder keinen Zweifel daran, dass Kochen ein Handwerk ist, das sich zur professionellen Expertise steigern lässt. Bei allen Variationen der Regionalküchen im Detail sind die massenmedialen Kochformate insofern transkulturell und transnational, als sie die Praxis des Kochens als kreativen wie für die Akteure anforderungsreichen Prozess ersichtlich machen (d. h. auch: als einen Prozess, an dem man Scheitern kann), wobei in vielen Formaten eben diese Dimension durch die Verknappung von Ressourcen (Zeit, Zutaten) dramaturgisch betont wird.

\subsection{Anschauungswissen}

Weitere Anreize entfalten die Koch-Formate, indem sie - zumindest potentiell ein vertieftes Verständnis des Kochens vermitteln. Während etwa Kochbücher einzelne Handlungsschritte im Unklaren lassen (müssen), explizieren die bewegten Bilder in einer sprachlich kaum zu erreichenden Präzision und Detailfülle die einzelnen Tätigkeiten und deren Phasenverlauf (u. a. das implizite Koch-Wissen der Akteure). Die dramaturgische Berücksichtigung dieses Sachverhalts variiert mit den Formaten. Während ein Format auf sprachliche Kommentierungen verzichtet und sich ganz auf die Kompetenz des Sehsinns verlässt („Silent Cooking“), heben andere Formate über eine gleichsam didaktische Kameraführung punktuell auf die Konstruktion von Anschauungswissen ab. Diese latente Funktion der visuellen Kommunikationen ist umso attraktiver, als sich die Aufmerksamkeit der Rezipienten in Abhängigkeit zu dem je eigenen Wissen und den je eigenen Interessen auf verschiedene Aspekte des Gezeigten richten kann.

\subsection{Imaginierte Vergemeinschaftung}

Die imaginierte Teilnahme am bildlich dargestellten Geschehen ist ein gewöhnlicher Rezeptionsmodus televisueller Realität. Neben und mit dem ,Realismus` der technischen Bildmedien spielen auch im Falle der Koch-Formate verschiedene Verstärkungsmechanismen der Ingangsetzung ,parasozialer Interaktion“ (Horton/ Wohl 1956) eine Rolle. Mittel unter anderen sind die direkte Ansprache der Rezipi- 
enten durch die Protagonisten oder eine Kameraführung, die die Fernsehzuschauer in Sequenzen ungeschnittener, real-time' am Geschehen teilnehmen lässt.

\subsection{Image-Arbeit}

Die Darstellungstechnik des Fernsehens sowie die verschiedensten inszenatorischdramaturgischen Eingriffe schaffen die idealen Voraussetzungen für die Beobachtbarkeit der Selbstdarstellungsformen der Akteure. Schon die bildliche Fokussierung der körperlichen Erscheinung bringt den anonym bleibenden (Bild-)Betrachter in eine besondere Position. Die in sozialen Situationen existierende Ordnung des Blickens ist für ihn prinzipiell außer Kraft gesetzt: Die Zuschauer können - zumindest im Bilderrahmen des jeweils Gezeigten - sehen, wohin sie wollen und so lange sie wollen, wobei insbesondere die „Mikroökologie des Gesichtsrahmens“ (Goffman 1969: 198) als Informationsmedium des Image-Geschehens fungiert. Die Koch-Formate schaffen über ihre Skripts dabei einen Unterhaltungswerte generierenden Rahmen, indem sie Images gefährden und dadurch forcierte Selbstdarstellungen bei den Akteuren erzeugen. ${ }^{6}$

Zusammenfassend kann man soweit feststellen: Die genannten Inszenierungsdimensionen tragen mit Hilfe der technischen Bildmedien zur Transnationalisierung der Formate bei, insofern sie Verstehen und Interesse (zumindest potentiell) in einem weitgehendem Verzicht auf kulturell spezifizierte Wissensbestände ermöglichen. Ja man könnte sagen: Die ,Kulturlosigkeit‘ der Formate auf dieser Ebene macht es möglich, dass sich die Kochsendungen als ein fester Bestandteil der Weltmedienkultur etablieren.

Das ist aber nur eine Seite der Medaille. Deren andere besteht darin, dass das Regionale, Nationale und Kulturelle implizit und explizit thematisiert wird. Einige der zentralen Inszenierungsdimensionen werden im Folgenden dargestellt.

\section{3. (Trans-)Nationalität, (Trans-)Regionalität und (Trans-)Kulturalität als Sinnressourcen der TV-Formate}

\subsection{Inszenierung von Nationalität}

Bemerkenswert ist zunächst, dass die Nation bzw. das Nationale verglichen mit der Region bzw. dem Regionalen von erheblich geringerer Bedeutung ist. Das gilt ins-

6 Mit einer drastisch zugespitzten Form des Wettkampfs arbeitet das Format „Gordon Ramsays Höllenküche“. Hier wird eine Kochschule als eine Art totale Institution inszeniert, in der die Kandidaten mit Denunziationen, Intrigen und Beleidigungen konfrontiert werden, die substantielle Imagearbeiten und Imagebeschädigungen sichtbar machen. 
besondere für die Identifizierung der verschiedenen Küchen. Obwohl die italienische, französische, deutsche oder amerikanische Küche gelegentlich Erwähnung findet, geht es im Detail also um die ligurische, bretonische, bayerische oder texanische Küche. Die gewöhnliche Verankerung der Identität von Küchen über regionale Bezüge schließt aber nicht aus, dass in Einzelfällen Nation als identitätsstiftende Klammer angesteuert werden kann. Die Bildmedien leisten hier wiederum wichtige Dienste. Mit dem Einsatz eines Zeichen- und Symbolrepertoires, dessen nationale Codierung im Alltagswissen als bekannt vorausgesetzt werden kann, lassen sich z. B. Interpretationsvoraussetzungen für die gewünschte Koch- und Küchennarration schaffen. Dabei ist festzustellen, dass das vorausgesetzte Alltagswissen selbst maßgeblich von den (Bild-)Medien generiert wird. Wenn z. B. für den britischen (Fernseh-)Koch Jamie Oliver „Jamies America“ als Bild- und Filmzusammenhang konstruiert wird, geschieht dies im Zugriff auf ein kollektives Bildgedächtnis, dessen Zeichen- und Symbole als bekannte (Stereo-)Typisierungen des ,Amerikanischen' bekannt sind: Cowboy-Outfit, rosafarbener Cadillac und das Bild des Highways, der sich geradlinig durch eine Wüstenlandschaft zum Horizont erstreckt, setzen als homologe Zeichen das Bild (Klischee) des (US-)Amerikanischen in Szene. Wie die im Einzelfall vorliegende Inszenierung ist das zugrunde liegende Sujet Resultat einer transnationalen Kulturindustrie, die - vom Roadmovie bis zum Werbe-Image - das ,Nationale' schon lange gerade auch als visuelle Schematisierung in globalen Märkten zirkulieren lässt.

Ein bemerkenswertes Beispiel bietet auch das japanische Format „Iron Chef“, das in einer Episode (der sogenannten „New York Battle“, Food Network 2000) einen Wettkampf der Nationen (Japan/USA) inszeniert. Das Nationale wird dabei in verschiedene Darstellungsmedien eingeschrieben, wobei neben den Handlungspraktiken der Protagonisten insbesondere die mediendramaturgischen Rahmungen einen Kampf der Nationalkulturen konstruieren. Als entsprechende Verstehensanweisung fungiert bereits der Vorspann, in dem die beiden Nationalflaggen in Feuerflammen verglühen, derweil dramatische (Militär-)Musik den Sinnrahmen des Bildes musikalisch unterstützt. Bemerkenswert ist die Verankerung des Geschehens im Nationalen umso mehr, als dieselbe für das japanische Format „Iron Chef“ gemeinhin keine Rolle spielt. Im Gegenteil - die Bezugnahme auf Küchen verschiedenster Weltregionen ist in diesem Format durchaus typisch. Das Nationale wird als Identitätsgenerator des Kochens und der Küche also nur in diesem dezidiert auf Vergleich und Wettbewerb abzielenden (Medien-)Spiel bemüht. Die Akteure sind dabei erkennbar in der Lage, Schemata bzw. (Stereo-)Typen aufzurufen, die als Attribute einer nationalstaatlichen Ess- und Koch-Kultur identifiziert werden können. So gibt der japanische Protagonist in der „New York Battle“ an, eine traditionelle japanische Speise zuzubereiten und kritisiert seinen US-amerikanischen Konkurrenten, der sich nach Fertigstellung des Gerichts (und vor dem Votum der Jury) mit erhobenen Armen in Siegerpose auf den Arbeitstisch stellt, im 
Hinweis auf kulturelle Unterschiede: Ihnen (den Japanern) sei das Schneidebrett heilig und das Verhalten seines Kontrahenten würde zeigen, dass er kein Meister seines Faches sei.

\subsection{Inszenierung von Regionalität und Tradition}

Insofern die technischen Bildmedien regionale Settings (Landschaften, Städte) und deren Bewohner zeigen können, führt ihr fotografischer Naturalismus zu einer scheinbar evidenten Einbettung von Kochpraktiken, Zutaten und Speisen in die jeweiligen Regionen. „Bizarre Food“ und „A Cook's Tour“ sind nur einige Beispiele eines Segments von Koch-Formaten im Stil der Reise-Reportage, für die die evidenzbasierte ,Beweisführung ${ }^{\natural}$ des Darstellungsmediums zentral ist. Muss hingegen auf das Zeigen von Kulissen verzichtet werden, ist eine regionale Semiotisierung bzw. Semantisierung von Speisen (Zingerle 1997) substantiell auf die Kommunikationsmedien Schrift und/oder Sprache angewiesen. Wie eine kulturelle Semantisierungen entlang regionaler Bezüge hergestellt wird, zeigt z. B. eine Episode des Formats „Alfredissimo“. Das Gespräch zwischen dem Gastgeber (Alfred Biolek) und seinem Gast dreht sich die Gesamtdauer der Sendung über im Wesentlichen um den umbrischen Landsitz des Gastes. Dieser berichtet in Passung zur jeweiligen Koch-Phase, dass die aktuell verwendeten Zutaten vom Garten dieses Landhauses stammen und illustriert diesen Sachverhalt z. T. mit kleinen Anekdoten. In den Erzählungen wird deutlich, dass das Rezept von einem benachbarten Bauern stammt (,unser Nachbar, ein einfacher, netter Bauer, hat uns einmal zum Essen eingeladen...") und offenkundig typisch für die Region ist. So entsteht im Laufe der Zeit das Bild einer, ursprünglichen Landschaft', für die dann letztlich der fertig zubereitete ,Schmortopf ${ }^{*}$ steht. Und das bedeutet auch: Der abschließende Probier-Genuss der Protagonisten speist sich aus einer imaginären Teilhabe an dem zuvor erarbeiteten semantischen Feld des, natürlichen Landlebens'. Es kommt also erst im Handlungsverlauf zu einer Bedeutungsvertiefung der Speise, die für den Zuschauer ohne diesen Kontext ein semantikfreies bzw. semantisch kontingentes Gebilde bliebe.

\subsection{Die Inszenierung von Transkulturalität}

Neben der (Re-)Produktion des Nationalen und Regionalen ist auch das Transnationale und Transkulturelle ein Reflexions- und Themenfokus televisueller KochFormate. Besonders deutlich wird das im Bereich der sogenannten, Cross-OverKüche', die durchaus zum Mainstream der massenmedialen Koch-Kultur gehört. In den verschiedensten Formaten wird die Zubereitung von Speisen vorgeführt, 
die von den gewählten Zutaten bis hin zur Präsentation der fertigen Gerichte als Mischung nationaler bzw. regionaler Küchen vorgestellt wird, wobei in der Untersuchungseinheit die Kombination von asiatischer und westlicher Küche am häufigsten vorkommt. Die Fernsehküche ist dann - insofern mit der Hochküche durchaus vergleichbar - eine Weltküche, indem sie einzelne Gerichte verschiedener Küchenkulturen integriert. Im Gegensatz zu Bildern eines andernorts (z. B. in dem massenmedialen Bereich der Nachrichten) stilisierten ,clash of cultures' erscheint das Kochen als ein Handlungsfeld, in dem nicht nur interkulturelles Verstehen und wechselseitige Akzeptanz, sondern auch die gegenseitige Wert- und Hochschätzung der Kulturen möglich, ja selbstverständlich sind. Sprachliche Kommentare, die die Qualitäten der Gerichte preisen, arbeiten dementsprechend oft mit Metaphern wie Gleichwicht, Balance und Harmonie.?

Obwohl die Vermischung regionaler Gerichte und Zubereitungsarten ein häufiger und deutlich sichtbarer Hinweis auf die transnationalen Verhältnisse der TVKüchen ist, liegt der entscheidende Indikator für den transnationalen und transnationalisierenden Charakter der Koch-Formate auf einer tieferliegenden, gleichwohl verdeckt bleibenden Ebene der Inszenierung. Gemeint ist der in allen Formaten unmissverständlich als zentral kommunizierte Leitwert des Geschmacks. Ihm sind die Kochhandlungen unterstellt und an ihm wird die Güte der Speisen ebenso wie die relative Könnerschaft der Akteure gemessen. Wie unter anderem Eva Barlösius und Wolfgang Manz (1988) gezeigt haben, ist die Entwicklung des Leitwerts Geschmack an verschiedene Modernisierungsprozesse gekoppelt, wobei neben und mit dem Umbau der stratifizierten zur funktional differenzierten Gesellschaft technische und ökonomische Entwicklungen eine besondere Rolle spielen: Indem die elaborierten Küchen im Laufe des 19. Jahrhunderts weniger den Distinktionsnotwendigkeiten der Oberschichten Rechnung tragen müssen, während sich zugleich größere Teile der Bevölkerung an einen über längere Zeitspannen anhaltenden Wohlstand gewöhnen, kann sich die ästhetische Dimension des Kochens von den bislang dominanten Funktionen emanzipieren und Geschmack als Eigenwert in den Mittelpunkt stellen. ${ }^{8}$

Unter diesem Gesichtspunkt kann man trotz aller Verschiedenheiten zwischen den Formaten von einer hochgradig homogenen Kochkultur der Massenmedien sprechen. Dies zeigt sich in besonderer Weise in denjenigen Formaten, in denen Kultur im Sinne eines aus, westlicher' Perspektive gänzlich anderen Weltverständnisses aufscheint, wie z. B. in den an der Reisereportage orientierten Fällen „Krasse Küche“ („Bizarre Foods“, USA) und „Eine Frage des Geschmacks“ („Anthony

7 So wird z. B. eine im Schweinebauchmantel gebratene Riesengarnele an Pfeffergurken nach abgeschlossener Dekoration vom Koch gepriesen als ,ein Gericht mit bayerischer Tradition, weltoffen präsentiert“ („Lafer: Einfach Kochen“, 05.12.07, EinsPlus).

8 Zu einer ausführlichen Rekonstruktion dieser Entwicklungen im Rahmen der Weberschen Theorie der Rationalisierung siehe Barlösius/Manz 1988: insb. 740-744. 
Bourdain: A Cook's Tour", USA). Hier kann sich segmentäre Gesellschaftsdifferenzierung (Geschlecht, Familie) als Ordnungsprinzip der Speisenzubereitung und des Essens andeuten. Während die Bilder gleichsam im Hintergrund eine Kultur jenseits des Geschmacksprinzips als Formengenerator der Küche erscheinen lassen, beziehen sich die im Vordergrund positionierten Protagonisten mit ihren Kommentaren und ihrem nonverbalen Ausdrucksverhalten jedoch nicht auf diese Sinnstrukturen, sondern auffällig häufig und auffällig deutlich auf die Geschmacksqualitäten der zubereiteten Speisen. ${ }^{9}$

$\mathrm{Ob}$ und inwiefern die Durchsetzung von Geschmack als Eigenwert der Küchen in verschiedenen Weltregionen an „multiple modernities“ bzw. an eine „Vielfalt der Moderne“ (Eisenstadt 2000) gekoppelt ist, lässt sich im Blick auf die KochFormate nicht sagen. Deutlich ist aber, dass wir es (auch) hier mit einer Küche zu tun haben, die religiösen Zwängen (z. B. Fastengeboten, Speisenverboten) ebenso enthoben ist wie solchen einer stratifizierten Gesellschaft, deren Mitglieder ständischen Koch- und Essordnungen verpflichtet sind und die sich als solche (zumindest bislang) in der Realität der Massenmedien etabliert.

Vor dem Hintergrund der hier nur kursorisch dargestellten Inszenierungsebenen kann man hinsichtlich der Bedeutsamkeit der Koch-Formate als Medien der Transnationalisierung abschließend Folgendes feststellen: Sie sind insofern transnational und transnationalisierend, als die audiovisuellen und computerisierten Medien in neuartiger Weise als Verbreitungsmedien fungieren, die die jeweiligen Inhalte in den verschiedenen Weltregionen verfügbar machen. Das gilt zwar prinzipiell schon für die Schriftkultur (z. B. der historisch weit zurückreichenden Kochbücher), wird aber erst unter Bedingungen des Satellitenfernsehens relevant. Insbesondere unter den Bedingungen des Internet kommt es zu einer Entlokalisierung bzw. Transnationalisierung der medialen Thematisierung von Kochen und Essen und der Zugänglichkeit entsprechender Materialien.

Neben und mit der technisch ermöglichten Transnationalisierung kann man von Transnationalisierung auf der Ebene der Darstellungsformen, der kommunizierten Sinnhaftigkeiten und Semantiken sprechen. Als visuelle Kommunikationen entfalten die Kochformate eine Anreizstruktur, deren Verstehen weitgehend unabhängig ist von einem sozial, kulturell und gesellschaftlich geformten Hintergrundwissen. Die Gestaltwerdung von Speisen, die sinnlichen Anschauungsreize, das Handwerk, die dargestellte (und imaginierte) Gemeinschaft und nicht zuletzt die Selbstdarstellungsformen der Akteure sind Elemente einer filmischen Dramaturgie, die an tendenziell universal verfügbare Wissensbestände anschließen kann. So ist es

9 So z. B. dann, wenn der Protagonist von „Bizarre Foods“, Andrew Zimmern, auf Samoa zu Gast bei einer Großfamilie ist. Die an Statuspositionen geknüpfte Rollenverteilung bei der Zubereitung, Zuteilung und dem Verzehr der Speisen wird sichtbar, ist aber für die Dramaturgie des Films nicht bedeutsam - diese kümmert sich ganz um das Lob des guten Geschmacks der Speisen und die Frage, wie dieser zu Stande kommt. 
nicht erstaunlich, dass entsprechend geformte Koch-Formate selbst Elemente einer transnationalen Weltkultur sind und diese - wie zahllose andere Formate der Medien-Unterhaltungs-, -Werbungs- und -Nachrichtenkultur - selbst forcieren und formen.

Hinsichtlich der Thematisierung von (Trans-)Nationalität, (Trans-)Regionalität und (Trans-)Kulturalität auf der Ebene der Inszenierungen lässt sich sagen: In den massenmedialen Kochformaten fungieren Nationalität, Regionalität und Kultur im Sinne eines von bestimmten Gruppen praktizierten Brauchtums als Sinnressourcen zur Gestaltung unterschiedlicher Skripts für unterschiedliche Publika. Der Bedarf für diese Sinnressourcen, so kann man z. B. mit Rudolf Stichweh vermuten, hängt wiederum unmittelbar mit den Kommunikationstechniken zusammen, in denen sich (u. a.) die Koch-Formate darstellen. Diese Techniken entfalten eine spezifische Problemkonstellation, die darin besteht, dass mit zunehmenden globalen Interrelationen „der Bedarf an Adressen als Mechanismen der Lokalisierung in einem globalen Kommunikationssystem" (Stichweh 1999: 463) zunimmt. Im Kontext der Kochformate tritt dieses Problem entlang der durch die Massenmedien selbst zunehmend sichtbar gemachten Gleichzeitigkeit verschiedenster Kochpraktiken, Koch-, Küchen- und Esskulturen in Erscheinung. Indem diese in ihrer Vielgestaltigkeit, Polykontexturalität und Hybridisierung zunehmend in der öffentlichen Sphäre sichtbar werden, kommt es um so mehr auf Klassifikationssysteme und Zuordnungsmechanismen an, wobei sich Nationalität und Regionalität offensichtlich besonders anbieten. Die Regionalküchen stehen dabei als Archiv erinnerbarer Formen zur Verfügung, die für Modulationen und Neuerfindungen genutzt werden. Mit Eric Hobsbawm und Thomas Ranger (1992) könnte man auch sagen: Die Kochformate stehen in einer Tradition der „Erfindung der Tradition“ zu Zwecken der Schaffung von Anhaltspunkten für die jeweils vorgeführten Kochhandlungen. Das Bemühen von Jamie Oliver, die ,ursprüngliche' Spaghetti-Bolognese des 18. Jahrhunderts nachzuempfinden („Oliver’s Twist“, 05.11.08, RTL2) ist dabei nur ein Fall von vielen.

Die audiovisuellen Medien übernehmen in dem Prozess der transnationalen Adressenbildung eine doppelte Funktion: Sie stellen in dem skizzierten Sinne eine global verständliche ,Grammatik' bereit und bilden zugleich das Medium für singuläre Erscheinungsformen, die mit $\operatorname{Namen}^{10}$ versehen werden können und in dieser Kombination dazu beitragen, dass in der Realität der Massenmedien eine transnational verfügbare Ordnung des Kochens und Essens entsteht, die u. a. das Regionale und Lokale als Inszenierung (re-)produziert. Und in diesem Sinne wird man sagen können, dass die massenmediale Kochkultur einen nicht unerheblichen Bei-

10 Ein Beispiel ist die Namensgebung von Speisen, die sich mit einer ebenfalls namentlich eingeführten Adresse von Nationalstaatlichkeit identifizieren lässt - so die Vorstellung eines Gerichtes als „Japanese Crab Salad“ („Iron Chef“, Food Network 2000). 
trag zur Konservierung, Dokumentation und Fortschreibung des (einst) national oder regional definierten Brauchtums leistet.

\section{Literaturverzeichnis}

Barlösius, Eva/Manz, Wolfgang (1988): Der Wandel der Kochkunst als genussorientierte Speisengestaltung. Webers Theorie der Rationalisierung als Grundlage einer Ernährungssoziologie. In: Kölner Zeitschrift für Soziologie und Sozialpsychologie 40(4): 728-746

Engelhardt, Dietrich/Wild, Rainer (Hrsg.) (2005): Geschmackskulturen. Vom Dialog der Sinne beim Essen und Trinken. Frankfurt am Main: Campus

Eisenstadt, Shmuel N. (2000): Die Vielfalt der Moderne. Weilerswist: Velbrück

Glaser, Barney G./Strauss, Anselm L. (1967): The Discovery of Grounded Theory: Strategies for Qualitative Research. New York: Aldine Transaction

Goffman, Erving (1969): The Presentation of Self in Everyday Life. New York: Doubleday

Goffman, Erving (1971): Interaktionsrituale. Über Verhalten in direkter Kommunikation. Frankfurt am Main: Suhrkamp

Hobsbawm, Eric/Ranger, Thomas (Hrsg.) (1992): The Invention of Tradition. Cambridge, UK: Cambridge University Press

Horton, Donald/Wohl, Richard R. (1956): „Mass-Communication and Para-Social Interaction. Observations on Intimacy at a Distance“. In: Psychiatry 19: 215-229

Keppler, Angela (1985): Präsentation und Information. Zur politischen Berichterstattung im Fernsehen. Tübingen: Narr

Ketchum, Cheri (2005): The Essence of Cooking Shows: How the Food Network Constructs Consumer Fantasies. In: Journal of Communication Inquiry 29(3): 217-234

Luhmann, Niklas (1996): Die Realität der Massenmedien. Opladen: Westdeutscher Verlag

Stichweh, Rudolf (1999): „Kultur, Wissen und die Theorien soziokultureller Evolution“. In: Soziale Welt. Zeitschrift für sozialwissenschaftliche Forschung und Praxis 50(4): 459-470

Teuteberg, Hans Juergen/Neumann, Gerhard/Wierlacher, Alois (Hrsg.) (1997): Essen und kulturelle Identität. Europäische Perspektiven. Berlin: Akademie Verlag

Wierlacher, Alois (2005): Was heißt, die Augen essen mit? Kulturwissenschaftliche und naturwissenschaftliche Betrachtungen über die Rolle des Sehsinns beim Essen. In: Engelhardt/Wild (2005): 129-143

Zingerle, Arnold (1997): Identitätsbildung bei Tische: Theoretische Vorüberlegungen aus kultursoziologischer Sicht. In: Teuteberg et al. (1997): 69-86 